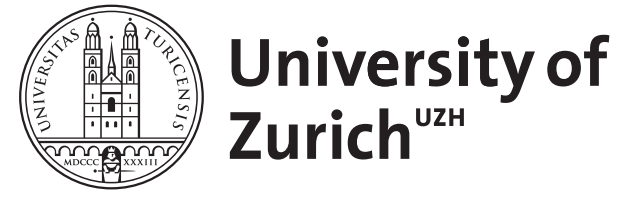

Ethische Gründe in der Konkreten Ethik

Halbig, Christoph

Posted at the Zurich Open Repository and Archive, University of Zurich

ZORA URL: https://doi.org/10.5167/uzh-98174

Book Section

Published Version

Originally published at:

Halbig, Christoph (2008). Ethische Gründe in der Konkreten Ethik. In: Halbig, Christoph; Kallhoff, Angela; Vieth, Andreas. Ethik und die Möglichkeit einer guten Welt : eine Kontroverse um die Konkrete Ethik. Berlin: De Gruyter, 82-92. 


\title{
Ethische Gründe in der Konkreten Ethik
}

\author{
von Christoph Halbig
}

0. Die Theorie ethischer Gründe steht im Zentrum des Projekts der Konkreten Ethik. Sie kommt an mehreren für sie zentralen Stellen zum Tragen, nämlich in der Theorie des Guten, des Sollens, in der Motivationstheorie, der Konzeption von Normativität und praktischer Vernunft sowie schließlich in der Frage nach der kausalen Wirksamkeit von Werten auf unser Handeln. Entsprechend entwickelt Ludwig Siep unterschiedliche Dimensionen seiner Theorie ethischer Gründe an ganz verschiedenen Stellen der Konkreten Ethik. Ich möchte im Folgenden den Versuch unternehmen, die verstreuten Bemerkungen zu diesem Problembereich, ausgehend von dem dafür zentralen Kapitel 2.1.3 „Gründe und Motive“, zu sichten und in einer Weise zusammenzuführen, die die Grundstruktur von Sieps Theorie ethischer Gründe sichtbar werden lässt. Gleichzeitig soll es diese Rekonstruktion erlauben, mögliche Kritikstrategien zu identifizieren und durch die Verortung der einzelnen Argumente im Gesamtzusammenhang von Sieps Theorie diese auf ihre Leistungsfähigkeit hin zu überprüfen.

Meine Rekonstruktion wird dabei geleitet durch die Unterscheidung von normativen und motivierenden Gründen: Normative Gründe sind die guten Gründe, die für eine Handlung sprechen, motivierende Gründe diejenigen, die uns tatsächlich zu einer Handlung (zumindest pro tanto) motivieren. Nicht alle guten Gründe, die wir für eine bestimmte Handlung haben, werden motivational wirksam (z. B. durch schlichte Unkenntnis), und nicht alle Gründe, die uns motivieren, sind auch gute Gründe (z. B. durch Irrtum). Praktische Rationalität zeichnet sich aber dadurch aus, dass es eben die guten Gründe sind, die für eine Handlung sprechen, die uns auch zu ihr motivieren. Damit ist zugleich dem Missverständnis vorgebeugt, dass es sich bei normativen und motivierenden Gründen um unterschiedliche Arten von Gründen handelt; ganz im Gegenteil geht es ja darum, einsichtig zu machen, wie ein Grund, der für eine Handlung spricht, diese auch motivieren kann - ein und dieselbe Entität bildet in diesem Fall den normativen und den motivierenden Grund für sie.

Diese für praktische Gründe aller Art grundlegende Unterscheidung gilt nun auch für den Bereich der ethischen Gründe, führt dort jedoch auf ganz spezifische Probleme: Die Frage nach der motivationalen Kraft stellt sich für ethische Gründe etwa in anderer Form als für prudentielle Gründe. Dass der Umstand, dass etwas zu meinem Wohlergehen beiträgt (prudentieller normativer Grund), 
mich dazu motiviert, in der entsprechenden Weise zu handeln, erscheint prima facie unproblematischer als dass dies bei dem Umstand, dass jemand ein Recht auf ein erhebliches Opfer meinerseits (ethischer normativer Grund) hat, der Fall ist. Es wird nun zu prüfen sein, welche konkrete Gestalt die Theorie ethischer Gründe, die Ludwig Siep in der Konkreten Ethik ausgearbeitet hat, aufweist.

1. In Bezug auf normative Gründe vertritt Siep eine werte-basierte Theorie: Im Anschluss an Hartmann und Broad behauptet Siep ein Primat des "ought to be“ vor dem „ought to do“ (KE 76). Das Tunsollen ergibt sich für Siep aus dem Bezug auf „den Erhalt bzw. die Beförderung des Guten, das sein soll“ (ebd.). Was sein soll ist nun für Siep, und darin liegt der holistische Charakter seiner Theorie, ein gutes Ganzes, das er mit dem Begriff der ,Welt' fasst. Als gutes Ganzes versteht er „im umfassenden Sinne die Verfassung bzw. Zustände der Welt, in denen eine gerecht geordnete Mannigfaltigkeit von Formen, Arten und Individuen sich ausbilden und gedeihen kann.“ (KE 135) „Alle Verhaltensnormen“ (ebd., meine Hervorhebung, C.H.) müssen zur „Herstellung oder Bewahrung dieses Zustandes“ (ebd.) beitragen.

Das Gute soll sein, und dies wiederum fundiert Gründe, es hervorzubringen, zu bewahren, zu fördern, es bildet mithin die Quelle normativer Gründe. Siep wendet sich in diesem Zusammenhang entschieden gegen eine Reduktion des Evaluativen auf das Normative, wie sie etwa im Rahmen der von Thomas Scanlon vertretenen buck-passing theory of value vertreten wird: ${ }^{1}$ Dass das menschliche Leben wertvoll ist, bedeutet nach Siep und pace Scanlon nicht lediglich, dass wir Gründe haben, mit $\mathrm{ihm}$ in bestimmten Weisen umzugehen. Dass ihm eine solche evaluative Eigenschaft zukommt, stellt mithin keine façon de parler dar, die das Vorliegen von normativ autonomen Gesichtspunkten zusammenfasst, sondern bildet vielmehr selbst die Quelle der entsprechenden Gründe. Ich habe etwa Grund, das Leben zu achten, weil es wertvoll ist; dass es wertvoll ist, zeigt nicht bloß das Bestehen bestimmter Gründe wie den, es zu achten, an.

Ethische Gründe als eine Spezies normativer Gründe neben anderen (etwa prudentiellen) zeichnen sich dadurch aus, dass sie sich nicht aus partikularem Guten (etwa dem Wohlergehen des Handelnden), sondern aus dem Bezug auf die gute Welt in ihrer Ganzheit ergeben. Siep spricht sogar von einem „Seinsollen des ethisch (sic!) Guten" (KE 173), eine These, die er als die Verpflichtung des Menschen erläutert, „das (zu) tun, was zur Erhaltung eines guten Zustandes der Welt notwendig ist" (ebd.).

In dem Bezug auf das gute Ganze liegt indes nicht das einzige Distinktionsmerkmal, das ethische Gründe von anderen Arten normativer Gründe abzugrenzen erlaubt. Das zweite Distinktionsmerkmal erkennt Siep in ihrem normativen Gewicht.

1 Vgl. Scanlon 1998, S. 95-100. 
Ethische Gründe sind für Siep normativ ausschlaggebende Gründe: ${ }^{2}$ „Wenn das Gute das wahrhaft erstrebenswerte und gesollte Ziel des menschlichen Handelns ist, dann lässt sich nicht einsehen, wodurch ein ethischer Handlungsgrund überwogen werden könnte." (KE 86)

Vergegenwärtigt man sich diesen grundlegenden Theorierahmen, in dem Siep seine Theorie normativer ethischer Gründe expliziert, könnte sich allerdings der Verdacht regen, dass die Kategorie des Ethischen gleichermaßen (i) überdehnt und (ii) normativ überfordert wird:

Erstens nämlich scheint sich nahezu jede Handlung auf den guten Zustand der Welt auszuwirken - und sei es nur dadurch, dass eine Handlungsoption ausgeschlagen wird, die in Bezug auf das Ganze billigenswerter gewesen wäre als die tatsächlich ergriffene: „Jede Handlung muss in eine gute Welt passen." (KE 86 meine Hervorhebung, C.H.) Ob es neutrale Handlungen geben kann, wie etwa, in einem stoischen Beispiel, das Zählen der Haare auf einem Kopf, erscheint als zumindest fraglich; falls es sie gibt, würden sie jedenfalls einen vergleichsweise marginalen Sonderfall bilden. Der Bereich des Ethischen wäre damit weniger ein distinkter Bereich des Normativen neben anderen, als vielmehr ein Gesichtspunkt, der alle Bereiche des Normativen durchdringt: Wenn ich mich etwa dafür entscheide, mir einen schönen Abend zu gönnen, wäre dies eine prudentielle Erwägung, die aber ihrerseits immer im Horizont des Ethischen gesehen werden müsste, nämlich unter der Fragestellung, ob mein schöner Abend zu einem guten Zustand der Welt beiträgt oder nicht. Nun könnte man mit Recht einwenden, dass dieser Umstand nicht notwendig eine Überdehnung des Ethischen bedeutet, sondern lediglich dessen spezifischen Charakter reflektiert: Ethische Gesichtspunkte zeichnen sich eben dadurch aus, dass sie Gesichtspunkte anderer Art nach Kriterien der Fairness, Unparteilichkeit etc. kritisch zu reflektieren und in ihrem normativen Gewicht einzuschränken erlauben.

Zweitens aber sind ethische Gründe für Siep nicht nur allgegenwärtig, sondern auch normativ ausschlaggebend. Dies bedeutet aber, dass immer dann, wenn etwa ein persönliches Projekt den Zustand der Welt insgesamt weniger gut werden lässt als etwa eine Tätigkeit im Dienst der Gemeinschaft, all things considered (wenn auch vielleicht zumindest ein pro tanto-Grund dafür bestehen bleibt ${ }^{3}$ ) kein Grund mehr besteht, das persönliche Projekt zu verfolgen. An dieser Stelle droht die Gefahr

${ }^{2}$ Siep vertritt damit, in der Terminologie der analytischen Debatte, eine overridingness-These in Bezug auf das normative Gewicht ethischer Gründe, vgl. dazu Halbig 2007, Kap. 5.2.

${ }^{3}$ Die Frage - die Siep m. E. offen lässt - ist hier, ob die normative Kraft von Erwägungen, die dem ethisch Geforderten entgegenstehen, ganz aufgehoben wird (John McDowell hat hierfür den Begriff des Zum-Schweigen-Bringens (silencing, vgl. McDowell 1979, \$3) geprägt), oder ob sie bestehen bleibt, jedoch durch das ethisch Geforderte überwogen wird und daher dann auch im all things considered-Urteil als Ergebnis praktischen Überlegens nicht mehr zum Tragen kommen darf. 
einer Hyperethisierung im Sinne einer Überforderung des Handelnden durch die Forderungen der Ethik. Muss es nicht auch möglich sein, einmal ,Urlaub von der Ethik' im Sinne eines Beitrags zum guten Ganzen zu nehmen?

Siep könnte auf dieses Problem in einer dreifachen Weise antworten. Erstens finden sich bei ihm Formulierungen, die der Ethik die Rolle eines Negativ-Kriteriums zuschreiben:

„Es ist kein Grund sichtbar, der besagte, dass auch einmal Handlungen ausgeführt werden dürfen, die nicht in eine gute Welt passen." (KE 86, meine Hervorhebung, C.H.)

Dass eine Handlung nicht in eine gute Welt passt, bildet einen ausschlaggebenden Grund dafür, sie zu unterlassen. Nun scheint es aber eine ganze Reihe von Handlungsoptionen zu geben, die gleichermaßen in eine gute Welt passen und zwischen denen dann möglicherweise nach außer-ethischen, etwa prudentiellen, Kriterien ausgewählt werden darf.

In diese Richtung weist zweitens auch Sieps Betonung einer Pluralität von „Aspekte(n) des Guten“ (KE 86), die dem Handelnden Abwägungsspielräume eröffnen sollen. Das gute Ganze stellt eben eine abstrakte Kategorie dar, die ganz im Geist der Konkreten Ethik der Spezifizierung bedarf. Siep selbst verweist auf mögliche Wertkonflikte (etwa zwischen Freiheit und Gleichheit); es wäre zudem zu erwägen, ob die Konzeption des guten Ganzen mit der Existenz von inkommensurablen Werten (zwei Werte A und B sind dann inkommensurabel, wenn A weder weniger noch mehr noch genauso viel wert ist wie B) vereinbar ist. Wiederum würden sich ethikfreie Spielräume der Entscheidung zwischen verschiedenen Spezifizierungen des guten Ganzen sowie zwischen inkommensurablen Werten ergeben, die anderen normativen Gesichtspunkten gehorchen könnten.

Drittens aber wäre zu erwägen, ob Siep nicht auch Raum für Bereiche lassen müsste und könnte, in denen die handelnden Subjekte vom Druck ethischer Gesichtspunkte bewusst entlastet werden. Hier legt sich eine Parallele zu der von Siep ausdrücklich anerkannten Kategorie „unbedingt schlechter Handlugen“ (KE 81) nahe, die ganz unabhängig von ihren Folgen und ihrer Stellung im Ganzen als intrinsisch verwerflich zu betrachten sind. Siep spricht in diesem Zusammenhang ausdrücklich von einer „Einschränkung der Teleologie“ (ebd.) als Grundzug der Konkreten Ethik. Ganz analog wäre zu prüfen, ob es nicht auch, nunmehr im positiven Sinne, Raum für persönliche Projekte und etwa auch akteurs-relative Gründe im Kontext von sozialen Formen wie der der Familie geben sollte, deren Bezug auf das gute Ganze weniger in dem teleologischen Verhältnis der Beförderung bzw. Maximierung bestehen würde, als in dem einer intrinsischen Konstitutionsbeziehung. Der Rolle eines Individuums etwa in seinen persönlichen Beziehungen zu den von der Handlung Betroffenen Rechnung zu tragen, könnte auf dieser Grundlage selbst dann als intrinsisch gut gelten, wenn etwa im Sinne der zweiten gerade angestellten 
Überlegung der Abwägungsspielraum zwischen zwei Werten, die in einer Handlung realisiert werden können, im Hinblick auf deren Beitrag zur Hervorbringung eines guten Ganzen nur gering wäre. Gerade Sieps Metapher des in eine gute Welt ,Passens' könnte den Ansatzpunkt bilden für eine stärkere Berücksichtigung der unterschiedlichen „modes of moral responsiveness" (Christine Swanton) ${ }^{4}$, die neben der Maximierung bzw. Förderung etwa auch Liebe, Achtung, Wertschätzung und Kreativität umfassen. ${ }^{5}$ Die Erkundung der Potentiale, die sich an dieser Stelle ergeben, stellt m. E. ein zentrales Desiderat für die Weiterentwicklung der Konkreten Ethik dar.

2. Dem Problem motivierender ethischer Gründe nähert sich Siep ausdrücklich aus der Perspektive normativer ethischer Gründe - und zwar anhand der Frage, ob solche guten ethischen Gründe auch dann, wenn sie „nicht von Affekten und Eigeninteresse unterstützt werden, gleichwohl zum Handeln motivieren können." (KE 87) Diese Formulierung des Problems würde erwarten lassen, dass Siep nach der motivationalen Relevanz eben der Entitäten fragt, die ihm zufolge normative Gründe konstituieren, nämlich Sachverhalte (vgl. KE 85), im Falle ethischer normativer Gründe etwa der Sachverhalt, dass etwas einen Bestandteil der guten Welt im Ganzen bildet.

Sieps Kernthese in der Theorie motivierender Gründe lautet nun aber,

„dass die Einsicht in die Güte bzw. den positiven Wert eines Zustandes, auch wenn dieser in keiner Beziehung zu einem sonstigen Wunsch oder Interesse des Handelnden steht, in ihm unmittelbar die Absicht der Aufrechterhaltung bzw. Herbeiführung dieses Zustandes auslösen und vermittels dieser Absicht zum Handeln bewegen kann." (KE 88, meine Hervorhebung)

Nach Siep konstituiert also nicht etwa der gute Zustand selbst, sondern die Einsicht in ihn, also ein psychischer Zustand des erkennenden Subjekts, den motivierenden Grund. ${ }^{6}$ Die Handlungsmotivation durch „Einsicht in rationale Gründe“ (KE 88) hält Siep nicht für einen erklärungsbedürftigen Sonderfall, sondern für den Normalfall auch außerhalb des Bereichs der Ethik. Ebenso wie der Wissenschaftler sich durch Einsicht in die interne Logik seines Gegenstandsbereichs leiten lässt, lassen sich Menschen durch die Einsicht in das ethisch Richtige leiten. Die Beweislast dafür, dass in jedem dieser Fälle ein versteckter appetitiver Zustand des Subjekts

\footnotetext{
${ }^{4}$ Vgl. Swanton 2003, S. 2 f. und Kap. 2.

5 Folgerichtig warnt Siep davor, die Rede von „gut ,für` das Ganze“ auf ein konsequentialistisches Maximierungsgebot einzuengen. Vgl. KE 66.

${ }^{6}$ Siep beruft sich entsprechend ausdrücklich auf die seit der Antike verbreitete Überzeugung, „dass die Einsicht in einen für wahr gehaltenen Sachverhalt, auch in einen für richtig gehaltenen Handlungszweck, hinreichend sein kann, um zum Handeln zu motivieren." (Siep 2004, S. 348) Vgl. a. Siep 2004, S. 349: „die Einsicht in das, was in einer bestimmten Situation richtig bzw. gut wäre, stellt für sich gesehen bereits ein Handlungsmotiv dar."
} 
den eigentlichen motivierenden Grund bildet, liegt nach Siep bei dem Vertreter einer solchen Entlarvungs-Strategie (vgl. KE 91) und es gibt für ihn bisher keinen Anlass, sie als erfolgreich zu betrachten.

Gegenüber der antipsychologistischen Position, der zufolge der Sachverhalt selbst und eben keinerlei psychischer Zustand wie der einer Einsicht motivational wirksam wird, besteht Siep indes darauf, dass "die ,Reaktionsfähigkeit des wertenden Subjekts ... eben zur motivierenden Kraft des Wertes hinzu(gehört).“ (KE 93.) Auch wenn also appetitive Zustände des Subjekts nach Siep keinen notwendigen Bestandteil des motivierenden Grundes bilden, gilt dies sehr wohl für die Reaktionsfähigkeit des Subjekts, die Siep im Wesentlichen als Einsicht auffasst. Dabei konzediert Siep dem Antipsychologisten ausdrücklich, dass „Werteigenschaften von Gegenständen, Ereignissen etc. (...) handlungswirksam werden“ können - allerdings nur „vermittels der emotionalen oder rationalen Rezeption durch das Subjekt." (KE 148.) Die entsprechenden Einstellungen des Subjekts jedoch werden wiederum ausdrücklich als „,durchlässig für Eigenschaften dieser Welt" verstanden (ebd.).

In der Terminologie der gegenwärtigen analytischen Debatte könnte man Sieps Theorie motivierender ethischer Gründe insgesamt wohl zutreffend als Variante eines reinen Kognitivismus klassifizieren. ${ }^{7}$ Die Einsicht in das ethisch Richtige, verstanden als kognitive Einstellung, ist es, die zur Motivation einer entsprechenden Handlung hinreicht. Sieps reiner Kognitivismus wird jedoch durch zwei weitere Merkmale seiner Konzeption eingeschränkt:

Erstens vertritt Siep eine pluralistische Motivationstheorie: Dass etwa Wünsche zum Handeln motivieren können, steht für ihn außer Frage. Die Motivation durch kognitive Einstellungen steht für Siep neben der durch appetitive Einstellungen wie Wünsche; ${ }^{8}$ Siep vertritt also, wie man im Anschluss an Jonathan Dancy sagen könnte, eine hybride Motivationstheorie. In der Terminologie von Thomas Nagel ${ }^{9}$ würde also auch Siep zwischen motivierten Wünschen einerseits, nicht-motivierten Wünschen andererseits unterscheiden: Dass ich einen Wunsch nach einem kühlen Bier verspüre, bildet ein Beispiel für einen nicht-motivierten Wunsch, dass ich den Wunsch habe, jemanden zu entschädigen, weil ich zu der Überzeugung gekommen bin, ihm eine solche Entschädigung schuldig zu sein, bildet ein Beispiel für einen motivierten Wunsch. Nach Siep können offenbar beide Arten von Wünschen mo-

${ }^{7}$ Die elaborierteste Variante eines reinen Kognitivismus in der Motivationstheorie hat Jonathan Dancy in Dancy 1993, Kap. 1-3 vorgelegt (und inzwischen zugunsten einer antipsychologistischen Position aufgegeben, vgl. Dancy 2000, Kap. 4-6).

${ }^{8}$ Vgl. etwa KE 147: „Ich habe aber ... zu zeigen versucht, dass nicht nur Wünsche unser Handeln bewegen können, sondern auch wahre Einsichten theoretischer und evaluativer Art." (meine Hervorhebung, C.H.).

9 Vgl. Nagel 1970, S. 29, dazu die Diskussion in Halbig 2007, Kap. 1.2.3. 
tivierende Gründe bilden. In der Regel ist es nach Siep sogar ein „Motivgemisch“ (KE 90), das unserem Handeln zugrunde liegt und in dem die Einsicht in gute Gründe einhergeht mit anderen, etwa appetitiven Motiven. Entscheidend ist aber, dass für Siep die Einsicht in solche Gründe in vielen Fällen und insbesondere in dem ethischer Erwägungen auch für sich genommen zur Handlungsmotivation hinreichend sein kann; sie ist also nicht notwendig auf andere Arten von Motiven angewiesen, auch wenn diese etwa zur Stabilisierung von Verhaltensmustern einen wichtigen Beitrag leisten können.

Zweitens ist die von Siep vertretene Konzeption praktischer Vernunft bewusst integrativ: Er versteht praktische Vernunft insgesamt wiederum als „Offenheit für das an sich Wertvolle" (KE 178), also gerade aus ihrem Bezug auf evaluative Merkmale der Wirklichkeit. Ihre Hauptaufgabe besteht darin, „das ,ought to be' in das ,ought to do" “ (ebd.) zu übersetzen. Praktische Vernunft ist für Siep gerade keine reine Vernunft, die Normativität in kantischer Tradition aus sich selbst generiert, sondern vernehmende Vernunft, die dem Subjekt dazu verhilft, sich der praktischen Dimension der Wirklichkeit zu vergewissern und ihr im eigenen Handeln Rechnung zu tragen. Die so verstandene Vernunft darf im Sinne von Siep zudem nicht ihrerseits isoliert und etwa durch Gleichsetzung mit dem Vermögen zu bloß kognitiven Leistungen den anderen Leistungen des Subjekts entgegengesetzt werden. Vielmehr schlägt Siep vor, sie eher adverbial zu verstehen als einen „Modus des menschlichen Erkennens, Fühlens, Wollens." (KE 179, meine Hervorhebung, vgl. a. Siep 2004, S. 352 f. und S. 357; S. 359 spricht Siep sogar von praktischer Vernunft als „eher ein Modus aller seelischen Kräfte"). Auch Emotionen etwa bilden mithin, insofern sie uns über die Wirklichkeit informieren, eine Dimension dieses integrativen Vernunftverständnisses. Ob auch der reine Kognitivismus in der Theorie motivierender ethischer Gründe über diesen integrativen Vernunftbegriff tatsächlich abgemildert wird, hängt natürlich davon ab, welche Ontologie etwa der Emotionen Siep vertritt; sollte es sich dabei z. B. in stoischer Tradition um einen reinen Kognitivismus handeln, würde die Einbeziehung von Emotionen in den Vernunftbegriff mit einem strikten Kognitivismus durchaus vereinbar bleiben. Sowohl die ausdrückliche Erwähnung etwa von Gefühlen, aber auch Sieps Verankerung in einer im weiten Sinne aristotelischen Tradition ${ }^{10}$ sprechen aber dafür, dass nach Siep die motivierende Rolle der Vernunft nicht in eins fällt mit der von kognitiven Einstellungen in einem eng gefassten Sinne.

Sowohl der pluralistische Ansatz von Sieps Theorie motivierender Gründe als auch ihre Verankerung in einer integrativen Theorie praktischer Vernunft führt auf einen erheblichen Klärungsbedarf, der freilich nicht mehr in den Bereich der Ethik

\footnotetext{
${ }^{10}$ Vgl. a. Sieps wiederholtes Plädoyer gegen eine „strikte Trennung rationaler von anderen Seelenvermögen." (KE 94) und Kap. 3.5.
} 
fällt und damit auch nicht in den Gegenstandsbereich der Konkreten Ethik im engeren Sinne gehört. So müsste etwa geklärt werden, wie sich motivierende Gründe, die allein aus kognitiven Einstellungen bestehen, zu Gründen, die nur oder auch aus appetitiven Einstellungen bestehen, verhalten. Gibt es bestimmte Arten von motivierenden Gründen, die durch ontologisch distinkte Entitäten konstituiert werden (zeichnen sich etwa ethische Gründe dadurch aus, dass für sie kognitive Einstellungen notwendig und hinreichend sind, während prudentielle Gründe notwendig auf Wünsche oder andere appetitive Einstellungen angewiesen sind, um motivational wirksam zu werden?), oder können selbst ethische motivierende Gründe, also Gründe ein und derselben Art, sowohl durch reine Überzeugungen als auch durch bloße (ethische) Wünsche als auch durch Paare von beiden konstituiert werden? Weiter wäre zu klären, ob der Erweiterung des Vernunftbegriffs auf ,alle seelischen Kräfte' (vgl. o.) nicht doch Grenzen gesetzt sind, etwa durch die Verbindung praktischer Vernunft mit praktischem Überlegen - die Orientierung an Gründen in einem solchen Prozess erscheint zumindest als paradigmatischer für das Ausüben praktischer Rationalität als etwa die nur indirekt und durch lange Übung mögliche Kultivierung etwa von Gefühlen (feelings). Der erweiterte Vernunftbegriffläuft zumindest Gefahr, dann doch wiederum die Einführung eines Vernunftbegriffs im engen Sinne erforderlich zu machen, was die Komplexität der Gesamttheorie auch in Bezug auf ihre rationalitätstheoretischen Konsequenzen erneut steigern würde.

3. Abschließend möchte ich mich aber auf eine, wie mir scheint, fundamentale Schwierigkeit konzentrieren, die sich aus der Gesamtstruktur von Sieps Theorie ethischer Gründe ergibt: Die Entitäten, die gute Gründe konstituieren, sind nämlich nach Siep prinzipiell für die Rolle motivierender Gründe ungeeignet. Bei ersteren handelt es sich ja nach Siep um Sachverhalte, bei letzteren um mentale Einstellungen der Einsicht in solche Sachverhalte. Natürlich ist die Einsicht in einen Sachverhalt aus erstpersönlicher Perspektive transparent auf das, was da jeweils eingesehen wird - was mich motiviert, ist der Sachverhalt, dass etwas meine Pflicht ist, nicht der Sachverhalt, dass ich eine Überzeugung mit dem Inhalt habe, dass etwas meine Pflicht ist. ${ }^{11}$ Dies ändert aber nichts daran, dass für Siep der Sachverhalt, der den guten Grund für eine Handlung darstellt, nur zusammen mit der „Reaktionsfähigkeit“ oder „Einsicht“ des Subjekts den motivierenden Grund für diese Handlung bilden kann. Die Möglichkeit, aus guten Gründen zu handeln,

11 Wenn Siep davon spricht, dass ein „Grund ... zum Motiv werden (kann), ohne dass er notwendig durch andere Motive als die Überzeugung von seiner Richtigkeit gestützt werden muss“ (KE 83) ist dies zumindest missverständlich. Jemand der überzeugt ist, dass p, hält p ipso facto für richtig es bedarf keiner weiteren Überzeugung zweiter Ordnung über die ursprüngliche Überzeugung mit dem Inhalt, dass diese richtig ist, noch auch wäre eine solche Überzeugung zweiter Ordnung in der Regel (von Sonderfällen abgesehen, in denen es um Fragen der Aufrichtigkeit, skeptische Anfechtungen etc. geht) ein geeignetes Handlungsmotiv. 
die aus der Perspektive des Handelnden konstitutiv für praktische Rationalität ist, wird mithin aus der philosophischen Beobachterperspektive Sieps in Frage gestellt. Wer meint, dass ihn der Sachverhalt motiviert, dass etwas seine Pflicht ist, irrt insofern, als das, was ihn tatsächlich motiviert, der Sachverhalt zusammen mit der Einsicht in ihn ist. ${ }^{12}$ Sowohl humeanische wie konsequent antipsychologistische, werte-basierte Theorien normativer Gründe sind hier demgegenüber in der Lage eine einheitliche Konzeption vorzulegen - in dem einen Fall sind es Wünsche, in dem anderen Werte, die gleichermaßen gute Gründe fundieren und zum entsprechenden Handeln motivieren.

Was würde gegen eine solche Vereinheitlichung von Sieps Theorie ethischer Gründe durch die Verbindung seiner werte-basierten Theorie normativer Gründe mit einer antipsychologistischen Theorie motivierender Gründe sprechen, die nicht nur den Vorteil theorieinterner Geschlossenheit, sondern eben auch den einer Übereinstimmung mit dem erstpersönlichen Selbstverständnis des handelnden Subjekts mit sich bringen würde?

Dagegen kann m. E. nicht sprechen, dass antipsychologistische Theorien strukturell nicht in der Lage wären, zu erklären, warum gute Gründe durch das Subjekt zumindest erkannt werden müssen, bevor sie motivational wirksam werden können - mit der von Dancy in diesem Zusammenhang eingeführten Kategorie der ,ermöglichenden Bedingung' (enabling condition) - hier für die motivationale Wirksamkeit des guten Grundes - etwa lässt sich dieser Schwierigkeit begegnen. ${ }^{13}$ Dagegen spricht weiter m.E auch nicht, dass ein Grund, wenn er ein Grund für mich sein soll, in einer bestimmten Beziehung zu mir stehen muss - dass ich einen Grund habe, jemanden aus schwerer See zu retten, ist etwa davon abhängig, dass ich schwimmen kann, kein besserer Retter zur Verfügung steht etc. All diese Gesichtspunkte, die mitbestimmend sind für die normative Relevanz, die der Sachverhalt, dass jemand zu ertrinken versucht, für mich hat, gehören jedoch zu den konstitutiven Bedingungen des normativen Grundes, nicht zu der Frage nach seiner motivationalen Rolle. Schließlich spricht auch nicht dagegen, dass eine antipsychologistische Motivationstheorie die Kultivierung der kognitiven und appetitiven Anlagen des Subjekts im Rahmen historischer Erfahrungsprozesse, auf die Siep großes Gewicht legt, erübrigen würde. Die Realität in ihrer normativen Relevanz in den Blick nehmen und ihr im Handeln Rechnung tragen zu können, setzt solche Leistungen gerade voraus - nicht als ein Addendum psychischer Einstellungen, das notwendig ist, um motivierende Gründe zu konstituieren, sondern als Voraussetzung dafür, dass das Erkannte selbst motivational wirksam werden kann.

\footnotetext{
${ }^{12}$ An dieser Stelle müsste indes zusätzlich präzisiert werden, ob Siep den Sachverhalt als etwas, das in der Welt der Fall ist, im Auge hat, oder lediglich den Sachverhalt qua intentionales Objekt der Einsicht. Auf der Grundlage der KE fällt es schwer zu entscheiden, was von beidem gemeint ist.

13 Vgl. dazu, aber auch zu den dadurch aufgeworfenen Problemen Dancy 2000, S. $127 \mathrm{ff}$.
} 
Es bildet einen zentralen Bestandteil der platonischen Tradition in der Ethik, dass es ungemein schwierig sein kann, der Realität standzuhalten. ${ }^{14}$ Ein Grund dafür ist aber aus Sicht des Antipsychologisten darin zu sehen, dass sie motivational gerade nicht neutral ist und eines zusätzlichen psychischen Elements bedarf, um in dieser Weise wirksam zu werden, sondern sich mit ihrem normativen Anspruch auch in ihrer motivationalen Kraft zur Geltung bringt.

Dass uns die guten Gründe, die wir haben, auch zum Handeln motivieren können, und dass zwischen der philosophischen Beobachterperspektive und der Teilnehmerperspektive des Handelnden keine systematischen Brüche entstehen, würde sicher selbst zu einer Welt beitragen, die als gut im Ganzen anzusprechen wäre. Das praktisch Wünschenswerte fällt natürlich nicht notwendig mit dem theoretisch zu Rechtfertigenden zusammen. Die konsequente Abwendung von einer psychologistischen Motivationstheorie scheint mir indes geeignet, dem Projekt der Konkreten Ethik erhebliche innere Spannungen zu ersparen, ihr zu einer größeren Geschlossenheit und intuitiven Plausibilität zu verhelfen und schließlich ihren Ansatz bei der „Idee einer guten Gesamtordnung der Welt“ (KE 74) als Ausgangspunkt der Ethik durch eine einheitliche Theorie praktischer Gründe zu stärken.

\section{Literatur}

Dancy, Jonathan 1993: Moral Reasons, Oxford: Blackwell.

Dancy, Jonathan 2000: Practical Reality, Oxford: Oxford Univ. Pr.

Halbig, Christoph 2007: Praktische Gründe und die Realität der Moral, Frankfurt a. M.: Klostermann.

McDowell, John 1979: Virtue and Reason, in: Monist 62, S. 331-350.

Murdoch, Iris 1970: The Sovereignty of Good, London, New York: Routledge und Kegan Paul. Nagel, Thomas 1970: The Possibility of Altruism, Oxford: Clarendon Pr.

Scanlon, Thomas M. 1998: What We Owe to each Other, Cambridge: Belknap Pr.

Ludwig Siep 2004: Vernunft und Tugend, in: Abwägende Vernunft, Praktische Rationalität in historischer, systematischer und religionsphilosophischer Perspektive, hrsg. v. Franz-Josef Bormann und Christian Schröer, Berlin, New York: de Gruyter, S. 344-360.

Swanton, Christine 2003: Virtue Ethics, Oxford: Oxford Univ. Pr.

14 Dazu Murdoch 1970, S. 64 f. 